\title{
Distributed On-demand Routing for LEO Satellite Systems
}

\author{
E. Papapetrou, S. Karapantazis, F.-N. Pavlidou
}

\begin{abstract}
Notwithstanding the limited commercial success of the first narrowband Low Earth Orbit (LEO) satellite systems, the interest of the scientific community in this type of systems has been revived on the basis of the current trend toward the migration to all IP-based services. LEO systems can play a pivotal role in providing services to areas where there is no substantial terrestrial infrastructure. Above all, LEO satellite systems can be used as backbone networks to interconnect autonomous systems worldwide. Such an approach provides flexibility in managing the resulting integrated network infrastructure and supporting innovative applications. In this context, routing data from the source all the way to the destination constitutes a daunting challenge. In this paper, a location-assisted on-demand routing (LAOR) protocol is proposed and evaluated. The proposed protocol introduces for the first time in satellite systems the concept of on-demand routing. However, its implementation is tailored to the requirements imposed by the characteristics of the topology of LEO satellite systems. The performance of the LAOR protocol is assessed for different link-cost metrics and compared to the one of centralized routing protocols proposed in the literature so far. Simulation studies further document and confirm the positive characteristics of the proposed protocol.
\end{abstract}

routing, on-demand, satellite, Low Earth Orbit

\section{Introduction}

Ubiquitous and pervasive communication is one of the major challenges that state-of-the-art networking faces nowadays. Satellite systems have always been the prevailing candidate for realizing the vision of anywhere, anytime connectivity on account of their inherent ability to provide global coverage. In fact, satellite networks have been used for years for a wide spectrum of services such as remote sensing, telephony services and television broadcasts [1]. In the past few years of the previous decade, the attention of the telecommunications community was drawn toward systems deployed at altitudes between $500 \mathrm{~km}$ and $1500 \mathrm{~km}$, which are termed low earth orbit (LEO) satellite systems [2]. LEO satellite systems provide low propagation delay which enables real-time communications as well as higher throughput compared to geostationary links. Furthermore, the low satellite altitude relaxes power requirements, allowing mobility and providing high data rates. Despite the advantages of LEO systems, the commercial success of the two operating narrowband LEO networks was limited, since at that time the networking market witnessed a worldwide proliferation of terrestrial infrastructure offering similar services. Satellite systems failed to compete with terrestrial networks in providing cost-effective broadband connectivity and as a result the interest in non-GEO systems temporarily dwindled away. Over the last years this interest has been renewed on the basis that satellite systems should augment the operation of terrestrial networks rather than compete with them [3]. According to this concept, LEO constellations can be employed to unify far-flung groups of people and provide high-quality telecommunication services to areas where there is no substantial terrestrial infrastructure. Moreover, other application 
scenarios include situations where terrestrial infrastructure is out of order, for instance during emergency situations, or not adequate owing to temporarily increased communications demands.

Furthermore, the current trend toward the migration to all IP-based services opens new opportunities to LEO satellite systems [4]. The unprecedented growth of the Internet, along with the proliferation of multimedia workstations, has spurred and prompted the development of innovative applications with high bandwidth and low end-to-end delay requirements. In addition, providing broadband communication services in world-wide scale through terrestrial infrastructures is not a trivial task. It involves the integration of a plethora of networking technologies and the coordinated operation of numerous autonomous systems. Besides interoperability issues, the greatest disadvantage is the lack of flexibility in designing and implementing network-wide management decisions to support new services and applications. The Internet represents such an example. The deployment of IPv6, which relies on the consent of network operators worldwide, has never been completed. For the same reasons providing QoS through a suitable routing algorithm is not feasible. Another typical example is the advent of overlay networks. The basic concept is to provide connectivity at the transport layer to support network-wide, flexible applications. It is apparent that network-wide coordination and management is essential for providing diverse and quality services. To this end, LEO satellite networks may play a pivotal role by providing backbone connectivity to terrestrial systems. The limited number of satellites needed allows the provision of high-quality worldwide connections. Furthermore, the management of such networks provides flexibility in adapting the network to new user demands and applications.

In the aforementioned context, routing data from the source to the destination, through the LEO network, constitutes a daunting challenge. Many of the proposed LEO constellations make use of inter-satellite links (ISLs) to provide direct connectivity between adjacent satellites. The resulting celestial network resembles a mesh and represents the most interesting architecture from a routing viewpoint, thus all the studies in this field focused on constellations with ISLs. In addition to the resulting highly connected graph, other characteristics such as the network size, the discontinuous operation of ISLs, the propagation delay and the unbalanced loading of the celestial network, pose strict requirements to the design of efficient routing algorithms. Most of the proposed approaches so far involve a centralized scheme for calculating paths for all origin/destination pairs. In addition, they take advantage of the deterministic dynamics of this network topology in order to divide the system period into a number of time intervals during which the topology of the network is fixed.

In this paper we propose a location-assisted on-demand routing (LAOR) protocol for LEO satellite IP networks that employ ISLs. The protocol introduces the well-known concept of on-demand routing into satellite networks. LAOR can be viewed as a variant of the ad-hoc on-demand distance vector (AODV) routing algorithm [5], tailored to the requirements imposed by the characteristics of LEO networks' topology. The prime aim of the LAOR protocol is to minimize end-to-end delay and delay jitter, while keeping signaling overhead to a minimum at the same time. Toward this end, the path discovery process is invoked independently for each individual origin/destination pair. However, our algorithm capitalizes upon the deterministic characteristics of the LEO mesh architecture in order to restrict the number of satellites that are flooded with route request messages. The performance of the proposed protocol is evaluated for different link-cost metrics and compared to the performance of proposed centralized routing protocols. Simulation studies further document and confirm the positive characteristics of the proposed protocol.

The remainder of the paper is structured as follows. In section 2 the related work is presented and the motivation for the new protocol is discussed. Then, the LAOR protocol is delineated in section 3 , highlighting its main differences with centralized, proactive routing schemes. Section 4 is devoted to the description of the simulation model used for the assessment of the new protocol. In section 5 simulation results are presented and discussed, while concluding remarks are drawn in section 6 . 


\section{Related Work and Motivation}

The performance of routing protocols depends on their perception of the network status. The state of nodes and related links should be frequently collected in order to depict the current network status. Routing relies heavily on the frequency and accuracy of this procedure which in turn depends on the rate that the collected information varies. The common approach in the literature as yet has been to capitalize upon network dynamics and periodically evaluate the propagation delay in each ISL, considering this as the metric to be minimized in the route calculation process. In [6] a centralized routing scheme geared toward ATM-based LEO/MEO networks was proposed, which relies on the Dijkstra shortest path algorithm to compute the optimal path for any pair of satellites. Werner extended that scheme by introducing a heuristic metric for calculating the cost of each ISL in [7]. The proposed metric evaluates the permanence of ISLs as well as the expected incoming traffic based on the link's location with regard to the Earth's surface. A similar approach is represented by the Probabilistic Routing Protocol [8]. This protocol takes into account the propagation delay and connectivity of ISLs in order to minimize the number of re-routing attempts that occur due to the fact that one of the ISLs that make up the path is switched off. The methodology described so far, provides acceptable implementation complexity considering that the satellites' movement is deterministic. However, such schemes are not designed or not supposed to take into account traffic related metrics such as queueing delay.

Later research laid emphasis on this shortcoming, nevertheless, the periodic nature of updates has been maintained. Mohorcic et al proposed a centralized routing protocol that is also based on the Dijkstra algorithm in [9],[10]. That protocol makes use of a cost metric that takes into account both propagation and queuing delay measurements and combines them with appropriate weighting factors. A similar approach was adopted by Akyildiz et al when dealing with the issue of routing in multilayered satellite IP networks in [11]. Another useful approach presented in [12] treated the issues of both topological design and routing in LEO satellite systems using a finite state automaton (FSA). A modified Flow Deviation algorithm for inclined Walker delta LEO satellite systems was developed in [13]. The main function of the Flow Deviation algorithm is to split traffic into many different paths. However, in order to reduce the algorithm's complexity and make it applicable to LEO networks, the modified algorithm uses a limited number of paths. That algorithm was further amended in [14] in order to be functional in Walker star LEO constellations, which are characterized by the discontinuous operation of ISLs.

All the aforementioned algorithms use proactive route calculation which involves high overhead and is also deeply affected by the network's size and connectivity. All the proposed systems so far involve a celestial network consisting of some tens up to some hundreds of satellites, which gives rise to justified skepticism about the feasibility and overhead requirements of routing protocols. Moreover, bearing in mind the connectivity of adjacent satellites (normally 4-8 ISLs), the task of keeping the related overhead low can be questioned. The overall overhead is also subject to the frequency of updates. The latter depends on the rate of information exchanged by the protocol. For example, for a traffic-adaptive protocol the route table update interval should be chosen to capture traffic variations, which results in increased overhead. In the case of LEO satellite systems even the propagation delay of each link changes rapidly. Although update intervals of about $30 \mathrm{sec}$ (adopted so far in the literature) provide satisfactory adaptation to propagation delay variations, when adaptivity to traffic related metrics (i.e., queueing delay) is required, periodic updates may result in high overhead. In fact, thus far no study has evaluated the related overhead of proposed routing algorithms in satellite systems.

Another major disadvantage of the proposed algorithms stems from their centralized implementation. Shouldering the burden of route calculation to a single point brings about some significant disadvantages such as reliability. In addition, the delay involved in collecting routing information and distributing routing 
decisions degrades network performance. Ekici et al [15] proposed a distributed routing scheme that routes each IP packet independently and induces no signaling overhead. That algorithm evaluates only propagation delay. Nonetheless, to avoid congested links, the algorithm introduces a queue size threshold over which packets are deviated to less congested routes.

The key concept of the strategy proposed in this work is to obviate the need for periodic route computation in highly connected and of great size networks such as LEO constellations, in order to minimize routing overhead. Furthermore, proactive methods have an impact on algorithm accuracy, since they can only provide an estimation of the optimal path for each source-destination pair during a specific interval. This estimation relies on the information about the network state collected in the last computation. The more frequent network state changes are, the poorer the performance of the routing algorithm is.

Therefore in this paper we propose the use of on-demand routing in satellite networks. By performing on-demand path computation the proposed algorithm manages to capture the path state at the time needed. Another observation supporting the on-demand approach refers to the non-uniform loading of the celestial network as a result of population dispersion, economic flourish and technology penetration diversity. Consequently routing requirements vary across the entire network, rendering the pre-computation of routes for all source/destination pairs inefficient. The key concept lies in allowing the routing algorithm to adapt to routing requests. On-demand routing is well-known in other types of networks, such as ad-hoc networks [16], however its suitability and applicability to satellite networks has never been addressed. The major drawback of such an approach is the flood-based mechanism used for discovering paths. The high connectivity degree of satellites may well lead to severe overhead requirements. However, the proposed algorithm capitalizes on the deterministic dynamics of the celestial network geometry to introduce the concept of restricted route request area. Hence, the effective area in which a route request is sent out is minimized along with the related overhead. Moreover, the size and the dynamics of the satellite network, impose severe modifications to mechanisms employed in traditional on-demand routing protocols, such as the maintenance of cached routes, the use of cached replies, the initiation of a path discovery and so on. Additionally, the high propagation delays and the non-uniform loading of a satellite network necessitate the use of both propagation and queuing delay as metrics for making routing decisions, contrary to the use of hop count in the case of traditional on-demand protocols in ad hoc networks. Finally, another key characteristic of the proposed protocol is its decentralized operation, providing increased flexibility and reliability.

\section{The Location Assisted On-demand Routing (LAOR) Protocol}

\subsection{System Model}

In this study we consider near polar (or Walker star) constellations that employ ISLs. Polar constellations present several attracting characteristics such as global coverage, simplified constellation management, deployment of ISLs, etc. In coherence to other studies we will study the most usual type of constellations where each satellite is assigned four ISLs: two intra-plane ISLs (namely, links to the adjacent satellites in the same orbital plane) and two inter-plane ISLs (that is, links to the neighboring satellites in the right-hand and left-hand orbital planes). While intra-plane ISLs are maintained for the whole satellite period, inter-plane ISLs are broken as satellites come close to the poles (figure 1) due to adverse pointing and tracking conditions and reestablished when satellites move to lower latitudes. Moreover, crossseam ISLs, namely links between satellites in counter-rotating orbits, are not used, as is the case with the Iridium system. In this paper we

are interested in routing within the network formed by satellites, called space segment. This network, on its abstraction level, can be modeled as a graph $G(V, E)$, comprising of a set of nodes $V$ and a set of edges $E$ (figure 2). It is clear that the size of $V$ is $|V|=N M$, where $N$ is the number of the orbital planes that the 


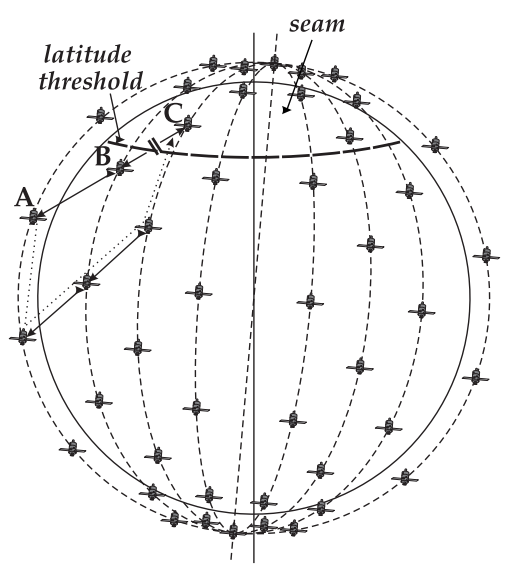

Figure 1: Example of typical polar constellation

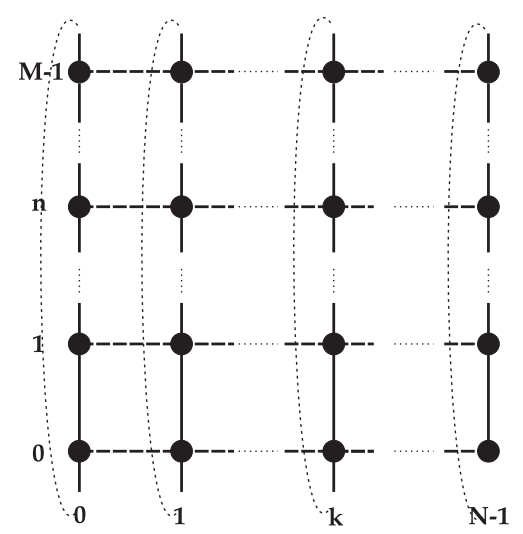

Figure 2: Resulting graph for typical polar constellation

system is comprised of and $M$ is the number of satellites per plane. In this graph each satellite is uniquely defined by the pair of virtual coordinates $(\bar{x}, \bar{y})$, where $\bar{x}$ and $\bar{y}$ denote the orbital plane and the position of the satellite in this plane respectively. Clearly, $\bar{x} \in[0, N)$ and $\bar{y} \in[0, M)$. The set of edges $E$ models ISLs connecting adjacent satellites. Consider an edge $\left\langle\left(\bar{x}_{k}, \bar{y}_{k}\right),\left(\bar{x}_{m}, \bar{y}_{m}\right)\right\rangle \in E$ connecting satellites $\left(\bar{x}_{k}, \bar{y}_{k}\right)$ and $\left(\bar{x}_{m}, \bar{y}_{m}\right)$. Then, for intraplane ISLs $\bar{x}_{k}=\bar{x}_{m}$ and

$$
\bar{y}_{m}= \begin{cases}\bar{y}_{k} \pm 1, & \text { if } \bar{y}_{k} \pm 1 \in[0, M) \\ \bar{y}_{k} \pm 1 \mp M, & \text { otherwise }\end{cases}
$$

while for interplane ISLs $\bar{y}_{k}=\bar{y}_{m}$ and

$$
\bar{x}_{m}= \begin{cases}\bar{x}_{k} \pm 1, & \text { if } \bar{x}_{k} \pm 1 \in[0, N) \\ \text { not } \quad \text { defined, } & \text { otherwise }\end{cases}
$$

It is clear from the previous equation that cross-seam ISLs are not considered in our study. The resulting graph $G(V, E)$ resembles a mesh network; however, ISLs are not permanent as mentioned before. To model this behavior, the availability of each edge $\left\langle\left(\bar{x}_{k}, \bar{y}_{k}\right),\left(\bar{x}_{m}, \bar{y}_{m}\right)\right\rangle \in E$ is determined by the value $a_{\left(\bar{x}_{k}, \bar{y}_{k}\right) \rightarrow\left(\bar{x}_{m}, \bar{y}_{m}\right)}$, which equals 1 when the latitude of both satellites $\left(\bar{x}_{k}, \bar{y}_{k}\right)$ and $\left(\bar{x}_{m}, \bar{y}_{m}\right)$ is less than the latitude threshold. This means that when either of the satellites resides within the polar regions, then $a_{\left(\bar{x}_{k}, \bar{y}_{k}\right) \rightarrow\left(\bar{x}_{m}, \bar{y}_{m}\right)}=$ $a_{\left(\bar{x}_{m}, \bar{y}_{m}\right) \rightarrow\left(\bar{x}_{k}, \bar{y}_{k}\right)}=0$. Furthermore, each edge in the graph $G(V, E)$ is characterized by the propagation delay

$d_{\mathrm{pr}_{\left(\bar{x}_{k}, \bar{y}_{k}\right) \rightarrow\left(\bar{x}_{m}, \bar{y}_{m}\right)}}$ of the corresponding ISL, as well as the queueing delay $d_{\mathrm{Q}_{\left(\bar{x}_{k}, \bar{y}_{k}\right) \rightarrow\left(\bar{x}_{m}, \bar{y}_{m}\right)}}$ encountered in

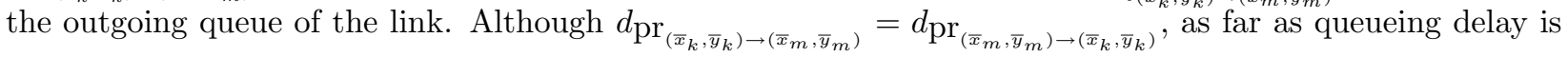
concerned, in general it holds that $d_{\mathrm{Q}_{\left(\bar{x}_{k}, \bar{y}_{k}\right) \rightarrow\left(\bar{x}_{m}, \bar{y}_{m}\right)}} \neq d_{\mathrm{Q}_{\left(\bar{x}_{m}, \bar{y}_{m}\right) \rightarrow\left(\bar{x}_{k}, \bar{y}_{k}\right)}}$ since the loading of ISLs is not the same in both directions.

\subsection{The Proposed Algorithm}

In this section the details of the LAOR protocol are spelt out, accentuating its main performance disparities in regard to periodic routing protocols proposed so far. The latter schemes are unable to capture variations in network state during successive updates. The new protocol aims to provide flexible adaptation to network state. To this end, it adopts an on-demand routing mechanism tailored to the requirements imposed by the topology of LEO satellite systems. The protocol consists of three processes: i) request area formation, ii) path discovery, and iii) route entry management. The path discovery process is independently invoked for each communication demand and aims at discovering the shortest path for an origin/destination pair. It is based 
on exchanging route request (RREQ) and route reply (RREP) messages, a technique extensively used in ad-hoc networks [16]. On-demand routing has been reckoned to provide the means of accurate estimation of the network state at the expense of routing overhead, produced by the usually flood-based nature of the path discovery process. However, to minimize the routing overhead, the proposed protocol capitalizes upon the predictable network topology, hence the name location-assisted on-demand routing (LAOR) protocol. The protocol instead of performing a network-wide search, prior to the initiation of the path discovery process, utilizes the request area formation process in order to minimize the space segment area into which a route request will be broadcasted. Finally, the route entry management process aims at managing stale routing information. Each process is discussed at greater length in the following subsections.

Since LAOR is an on-demand distance vector algorithm, each satellite $v_{i} \in V$ must maintain a monotonically increasing counter, called sequence number $\left(s e q_{i}\right)$. The concept of sequence number is well-known in dynamic routing algorithms ([16], [5]) and serves on the one hand to avoid loop formation and on the other to supersede stale cached routes. The meaningfulness of this counter will be revealed in more detail later on. Furthermore, each satellite should maintain a routing table $\left(R T_{i}\right)$ containing one entry for each destination in the network. The structure of each route entry includes the following information:

- $r t$ _dst: the address of the destination satellite.

- rt_dst_seqnum: the last heard sequence number from the destination satellite.

- rt_next_hop: the satellite to which a packet for the destination should be forwarded.

- rt_owner: this boolean field is true only if the route to rt_dst was discovered as a result of a route request initiated by this node. The field is used to indicate whether this node can use the route to reply to route requests from other nodes.

- rt_path_cost: the cost of the entire path, that is the sum of the ISLs' costs. In this study we consider that the cost of each ISL can be either its propagation delay or the sum of propagation delay and the queuing delay encountered by a packet in the ISL's outgoing queue.

- rt_path_expiration_time: the time instant at which the route entry and thus, the path, will become invalid due to the switching off of an interplane ISL. This time can be calculated on account of the constellation deterministic dynamics.

- rt_timestamp: the time instant at which the route entry was recorded.

In order to support the use of the last two fields of a route entry, each satellite maintains a clock. Networkwide synchronization of clocks is required and can be easily achieved on network set-up and maintained by the network manager.

In addition to the routing table, each satellite $v_{i} \in V$, maintains a table $R e q T_{i}$ where it stores information regarding route requests originated by other network nodes and seen by node $v_{i}$. The table contains one entry for each satellite in the network and each entry has the following fields:

- reqt_orig: the address of the satellite that originated a route request. The existence of the entry implies that a request originated by node reqt_orig was received by the current satellite in the past.

- reqt_seqnum: the last heard sequence number carried in a route request from satellite reqt_orig. As it will be explained later on, each route request is uniquely identified by the sequence number.

- reqt_cost: the cost from the originator of the request to the current satellite, recorded on the route request message which is identified by reqt_seqnum. 


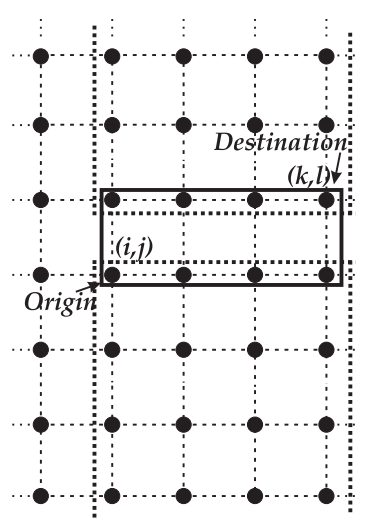

Figure 3: Request area formation $\left(w_{\min }=0\right)$.

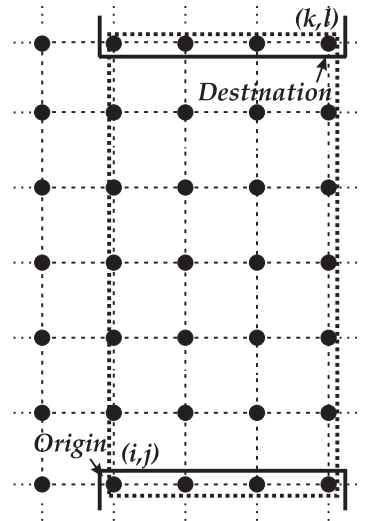

Figure 4: Request area formation $\left(w_{\min }=0\right)$.

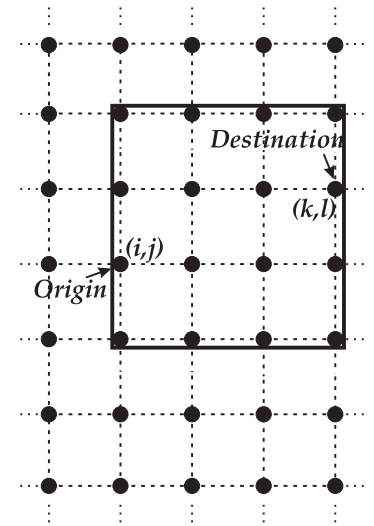

Figure 5: Request area formation $\left(w_{\min }=1\right)$.

- reqt_next_hop: the address of the satellite that forwarded the route request message. This field indicates a path to the originator of the request and is used for forwarding route reply messages back to the originator of a request.

The last field is necessary since for a given origin/destination pair, the forward and the reverse paths are not the identical in general. Take for example the case that queueing delay is used as the cost of a link. As mentioned in Section 3.1, in general $d_{\mathrm{Q}_{\left(\bar{x}_{k}, \bar{y}_{k}\right) \rightarrow\left(\bar{x}_{m}, \bar{y}_{m}\right)}} \neq d_{\mathrm{Q}_{\left(\bar{x}_{m}, \bar{y}_{m}\right) \rightarrow\left(\bar{x}_{k}, \bar{y}_{k}\right)}}$ because the loading of ISLs is not the same in both directions. Last but not least, each satellite has to maintain a queue, called LAOR queue. Since LAOR is an on-demand protocol, it uses this queue to temporarily store packets that are waiting for a path to be set up.

\subsubsection{Request Area Formation Process}

When a ground terminal initiates a connection with another one or when an already communicating terminal is handed over to a new satellite, the request area formation process is initiated by the serving satellite as soon as the up and down links are established and the first packet arrives. The process is initiated whether a route to the destination satellite exists or not. The latter strategy of ignoring the existence of a route to destination aims at taking advantage of traffic aggregation at satellites since each path discovery for a new terminal will also be of benefit to ongoing connections between terminals served by the same pair of satellites. The request area formation process constitutes the workhorse of the proposed protocol and aims to keep routing overhead to a minimum. This is accomplished by capitalizing on the deterministic topology of LEO satellite systems in order to diminish the area within which routing requests are sent out. The rationale behind this approach is, that given the origin/destination pair, the shortest path based on propagation delay lies within the smallest rectangle area defined by the communicating satellites, called from now on minimum route request area (the solid lines in figures 3,4 ). In fact, the shortest path based on propagation delay can be precisely calculated ([15]) using the deterministic dynamics of the topology. However, the minimum route request area provides a set of paths (including the shortest one based on propagation delay) with similar propagation delays. LAOR makes the observation that when queueing delay (which depends on the loading of links) is taken into account, there is a strong possibility that the optimal path is located within the minimum route request area, therefore it limits the broadcasting of route request messages within this region. Such an approach could be beneficial to the algorithm since it also provides the means for avoiding congestion by utilizing a set of paths rather than a single path to destination. 
Let us assume that a ground terminal served by the $j^{\text {th }}$ satellite in the $i^{\text {th }}$ orbital plane (hereafter referred to as the source satellite with virtual coordinates $\bar{x}_{s r c}=i$ and $\bar{y}_{s r c}=j$ ) wants to communicate with a ground terminal that is covered by the $l^{\text {th }}$ satellite in the $k^{\text {th }}$ orbital plane (from now on referred to as the destination satellite with virtual coordinates $\bar{x}_{d s t}=k$ and $\bar{y}_{d s t}=l$ ). According to the model described in Section 3.1, the request area formation process deals with the determination of a subgraph $G^{\prime}\left(W, E^{\prime}\right), W \subseteq V, E^{\prime}=E(W)$ of $G(V, E)$ for which the set $W$ consists only of the satellites that a route request message is allowed to reach. That is $W=\left\{\left(\bar{x}_{k}, \bar{y}_{k}\right) \mid\left(\bar{x}_{k}, \bar{y}_{k}\right) \in V, \bar{x}_{k} \in \bar{S}_{x}, \bar{y}_{k} \in \bar{S}_{y}\right\}$, where $\bar{S}_{x}$ and $\bar{S}_{y}$ are the sets of acceptable values for virtual coordinates of satellites in $W$. It is clear that $\bar{S}_{x}=\left[\bar{x}_{\min }, \bar{x}_{\max }\right]$ with $\bar{x}_{\min }=\min \left\{\bar{x}_{s r c}, \bar{x}_{d s t}\right\}$ and $\bar{x}_{\max }=\max \left\{\bar{x}_{s r c}, \bar{x}_{d s t}\right\}$. As far as $\bar{S}_{y}$ is concerned, there are two alternatives for defining the rectangle area containing the source and destination satellites (compare solid and dashed lines in figures 3,4). However we are interested in the minimum route request area therefore,

$$
\bar{S}_{y}= \begin{cases}{\left[\bar{y}_{\min }-w_{\min }, \bar{y}_{\max }+w_{\min }\right],} & \bar{y}_{\max }-\bar{y}_{\min } \leq\left\lfloor\frac{M}{2}\right\rfloor \\ {\left[0, \bar{y}_{\min }+w_{\min }\right] \cup\left[\bar{y}_{\max }-w_{\min }, M-1\right],} & \text { otherwise }\end{cases}
$$

where $w_{\min } \geq 1, \bar{y}_{\min }=\min \left\{\bar{y}_{s r c}, \bar{y}_{d s t}\right\}$ and $\bar{y}_{\max }=\max \left\{\bar{y}_{s r c}, \bar{y}_{d s t}\right\}$. The parameter $w_{\min }$ has the effect of expanding the calculated route request area (figure 5) and serves to guarantee that the subgraph $G^{\prime}\left(W, E^{\prime}\right)$ is connected, therefore a path to destination always exists. Consider for example the scenario depicted in figure 1. Suppose that satellite $A$ wants to establish a path with satellite $C$ and $w_{\min }=0$. Let us define $\Delta Y_{\bar{S}_{y}}=\bar{y}_{\max }-\bar{y}_{\min }$. In this case, since $\bar{y}_{s r c}=\bar{y}_{d s t}, \Delta Y_{\bar{S}_{y}}=0$. Satellite $C$ is located over the pole regions, thus the inter-plane ISLs between satellites $B$ and $C$ is switched off and it is not possible to set up a path between these two satellites. Nonetheless, when $w_{\min }=1$ a path can be established through satellites located at lower altitudes. This path is illustrated by the dashed line in figure 1. In general the problem arises due to the switching off of some interplane ISLs near the polar regions. Let us define the set $E_{i}^{\prime}$ of interplane ISLs that belong to the minimum route request area, are active and connect satellites of plane $i$ with satellites of plane $i+1$. Therefore, $E_{i}^{\prime}=\left\{\left\langle\left(\bar{x}_{k}, \bar{y}_{k}\right),\left(\bar{x}_{m}, \bar{y}_{m}\right)\right\rangle \mid a_{\left(\bar{x}_{k}, \bar{y}_{k}\right) \rightarrow\left(\bar{x}_{m}, \bar{y}_{m}\right)}=1, \bar{x}_{k}=i, \bar{x}_{m}=\bar{x}_{k}+1, \bar{y}_{k} \in \bar{S}_{y}, \bar{y}_{m}=\bar{y}_{k}\right\}$, $\forall i \in \bar{S}_{x}-\left\{\bar{x}_{\max }\right\}$. Clearly, $E_{i}^{\prime} \subseteq E^{\prime}$. For the graph $G^{\prime}\left(W, E^{\prime}\right)$ to be connected it must be,

$$
E_{i}^{\prime} \neq \emptyset, \forall i \in \bar{S}_{x}-\left\{\bar{x}_{\max }\right\}
$$

In other words, if at least one active interplane ISL exists in the minimum route request area for each pair of adjacent planes, then at least one path to destination can be established. The parameter $w_{\min }$ is the minimum value so that Eq. 4 is valid. The value of $w_{\min }$ depends on the constellation design that determines the availability of interplane ISLs, $a_{\left(\bar{x}_{k}, \bar{y}_{k}\right) \rightarrow\left(\bar{x}_{m}, \bar{y}_{m}\right)}$. However, $w_{\min }$ can be determined in advance. It must be noted at this point that $w_{\text {min }}$ only refers to the y direction of the route request area. To explain the reason for that, we refer again to the previous example of figure 1. The expansion of the route request area only in the $\mathrm{x}$ direction does not solve the problem of path existence between satellites $\mathrm{A}$ and $\mathrm{C}$ since it does not introduce any new interplane ISLs in the route request area. Therefore, the decision for not using $w_{\min }$ in the $\mathrm{x}$ direction serves the minimization of the routing overhead.

As mentioned before, when queueing delay is considered, there is the possibility that the shortest path does not reside in the minimum route request area. To diminish this possibility, a value width $>w_{\min }$ may be used in Eq. 3. This is because a wider search area, which is called restricted route request area, may provide longer paths in terms of propagation delay, but less congested, therefore shorter in terms of end-to-end delay. Notwithstanding this advantage, a wider route request area translates into increased signaling overhead. 


\subsubsection{Path Discovery Process}

After the formation of the restricted route request area, the path discovery process is initiated. The source satellite increases its sequence number and generates a route request ( $R R E Q)$ packet, which contains eight fields that are described below:

- $p k t_{-}$type_id: this field is used to indicate that this is a RREQ packet and therefore provides differentiation from other packet types that LAOR uses.

- $p k t \_s r c$ : this field contains the address of the source satellite.

- pkt_src_seqnum: this field contains the sequence number of the source satellite. Furthermore, it uniquely identifies a RREQ packet.

- $p k t_{-} d s t$ : this field contains the address of the destination satellite.

- $p k t$ dst_seqnum: this field contains the last heard sequence number from the destination satellite. If the source satellite does not have a route entry in table $R T_{i}$ for satellite $p k t_{-} d s t$, then this field is set to zero.

- pkt_path_cost: this field is used to record the cost of the path from the source satellite to the satellite that the packet has reached. When the packet reaches the destination, the field contains the total path cost from the source to the destination satellite.

- pkt_path_expiration_time: this field indicates the time instant at which the path, formed so far, will become invalid due to the switching off of an ISL.

- pkt_timestamp: this field contains the time instant at which the RREQ packet was sent out by the source satellite.

The RREQ packet will be sent to all the neighboring satellites that also belong to the restricted route request area. The sending satellite can derive the set of those satellites by verifying that for their virtual coordinates $(\bar{x}, \bar{y})$ holds that $\bar{x} \in \bar{S}_{x}$ and $\bar{y} \in \bar{S}_{y}$. During its travel toward the destination, a RREQ packet may reach: i) an intermediate satellite that does not have a valid route to the destination (Case $\boldsymbol{A}$ ), ii) the destination satellite itself (Case B) and/or iii) an intermediate satellite that has a valid route to the destination (Case $\boldsymbol{C})$. In the following it will be discussed how LAOR defines valid routes.

\section{Case $A$}

Suppose that an intermediate satellite does not have a valid route to the destination satellite. It first must decide whether to accept or not the RREQ packet. Such a packet is accepted only if its pkt_src_seqnum field is greater than the last known sequence number for the source satellite (this number is stored in the corresponding entry of table ReqT) or if the two numbers are equal and the RREQ packet has arrived through a shorter path than the previous copy of the same RREQ packet. This means that the pkt_path_cost field is smaller than the reqt_cost field of the ReqT entry concerning the source satellite. In any other case the RREQ is not acceptable and is suppressed. By using these criteria, LAOR avoids loops in the formation of the path. To make this clear consider the case that the same RREQ packet is received again by the same intermediate satellite. Then it will not be accepted because even if the sequence number is the same, the path cost will be greater since it increases monotonically. At this point it should be noted that accepting RREQ packets with the same sequence number serves the purpose of supporting path costs other than the total delay (propagation and queueing delay). Consider the case that the link-cost metric is calculated based 
only on the ISL's propagation delay. Then the first packet that arrives at the intermediate satellite has come through the minimum delay path but not necessarily through the minimum cost (propagation delay in this case). By accepting a delayed RREQ packet with the same sequence number we can discover the minimum cost route.

If the RREQ packet is accepted, then the intermediate satellite should update (or create if not existent) the entry of table ReqT that concerns the source satellite with the respective fields of the RREQ packet. The satellite that forwarded the RREQ is stored in the reqt_next_hop field of this entry. This serves to establish a reverse path to the originator of the RREQ packet that can be used by RREP messages. The reason for setting up this path is that in general a path toward the source satellite may not exist in the routing table $R T$. Furthermore, the reverse path is not the minimum cost path in the direction from the intermediate satellite to the source satellite. Thus it is not wise to store it in the routing table. After setting up the reverse route, the intermediate satellite updates the pkt_path_cost field and forwards a replica of the RREQ packet to the contiguous satellites (excluding the one that sent the RREQ) with virtual coordinates $\bar{x} \in \bar{S}_{x}, \bar{y} \in \bar{S}_{y}$ which also satisfy the following inequality

$$
\begin{gathered}
\bar{x} \geq \bar{x}_{c}, \quad \text { if } \bar{x}_{s r c} \leq \bar{x}_{d s t} \\
\text { or } \\
\bar{x} \leq \bar{x}_{c}, \quad \text { if } \bar{x}_{s r c}>\bar{x}_{d s t}
\end{gathered}
$$

where $\bar{x}_{c}$ denotes the $x$-coordinate of the intermediate satellite. No additional constraints are imposed for the $y$-coordinate, in order to guarantee the existence of at least one path, according to the rationale expressed at the end of Section 3.2.1.The aim of this inequality is to forward a RREQ packet only toward the direction of the destination satellite in order to keep overhead to a minimum. At this point it should be stressed that the intermediate satellite will not forward a RREQ obtained from a path that will become invalid very shortly. To decide whether a RREQ packet will be forwarded, the intermediate satellite uses the pkt_path_expiration_time field of the RREQ and validates the equation

$$
t \leq p k t \_p a t h \_e x p i r a t i o n \_t i m e-\text { expiration_threshold }
$$

where $t$ is the current time and expiration_threshold is a parameter of the LAOR protocol that determines the minimum acceptable lifetime of a discovered path. Only if this equation holds will the satellite forward the RREQ packet. The rationale behind this parameter is to avoid establishing a path that will become invalid very shortly due to the switching off of one of the ISLs that make it up. If the RREQ is finally forwarded, the intermediate satellite calculates the expiration time $t_{\text {exp }}$ of the link through which a replica of the RREQ will be forwarded. Then it updates the pkt_path_expiration_time field of the replica with $\min \left\{t_{\text {exp }}\right.$, pkt_path_expiration_time $\}$.

\section{Case $B$}

One case is that the RREQ will eventually reach the destination which has to decide (according to the procedure described in Case A) on whether the RREQ is acceptable or not. If so, the satellite increases its sequence number, updates ReqT and produces a RREP message that contains the following information:

- $p k t$ typeid: this field indicates that this is a RREP packet.

- pkt_src: this field contains the address of the satellite that produces the RREP (the destination of the RREQ or an intermediate).

- pkt_dst: this field contains the address of the source satellite that produced the RREQ. 
- pkt_dst_seqnum: this field is set equal to the sequence number of the satellite that produces the RREP. This number uniquely identifies the RREP message.

- pkt_path_cost: this field is set equal to the value written in the corresponding field of the RREQ packet, which is the cost of the path from the source satellite to the destination.

- pkt_path_expiration_time: this field communicates the path's expiration time instant to the source satellite and is set equal to the respective field of the RREQ packet.

- pkt_timestamp: this field is set equal to the corresponding field of the RREQ packet.

The RREP message is forwarded to the source of the route request by using the entries concerning the source satellite in the tables ReqT of the intermediate satellites. Upon receipt of a RREP, an intermediate satellite stores in its routing table $R T$ a path to the destination satellite according to the data carried on the RREP packet. However, the fields rt_path_cost and rt_expiration_time are not stored because the respective values carried in the RREP packet correspond to the path from the source to the destination satellite and not to the path from the intermediate satellite to the destination. Furthermore, the field pkt_owner is set to false to indicate that the satellite is an intermediate in the path. This means that the fields rt_path_cost and rt_expiration_time do not carry valid information and therefore can not be used for replying to other route requests. It must be noted at this point that if a route entry for the destination satellite already exists, then it is superseded only if the pkt_dst_seqnum carried on the RREP is greater than the rt_dst_seqnum of the existing entry or if the two numbers are the same and the path cost of the new path is smaller than the cost of the existing entry. Finally, the source satellite can commence data transmission as soon as the first RREP is received and can later update its routing information if it learns of a better route. It must be noted that in the route entry stored by the source satellite the field pkt_owner is set to true.

\section{Case $C$}

There is a possibility that a RREQ packet will produce a RREP before reaching the destination. This happens when it reaches an intermediate node that holds a valid route to the destination. In order for a route to be considered valid, its value rt_owner should be true. Furthermore, its rt_dst_seqnum field must be greater than the pkt_dst_seqnum contained in the RREQ packet and it must have been recorded after the time instant at which the RREQ was sent out (this is indicated by the pkt_timestamp field of the RREQ packet). The condition regarding sequence numbers is also used in other dynamic routing protocols such as AODV [5] and guarantees loop-free paths. The second condition is used to limit replies by intermediate nodes only to those having up-to-date routes. Furthermore, a route is considered valid if its lifetime is acceptable based on equation (6). In this case, the minimum of the time instant carried in the RREQ packet and the expiration time of the cashed route is used as path expiration time. After confirming that the RREQ packet can be accepted (by the same procedure as in case A), the intermediate satellite increases its sequence number and produces a RREP. The path cost carried in the the RREP packet is the sum of the cost carried in the RREQ packet and the cost of the stored route.

\subsubsection{Route Entry Management}

As mentioned earlier, the path's expiration time is stored in each route entry. The purpose of this information is to purge the route entry for the destination satellite before the time instant at which the route is considered to become invalid. Nonetheless, a new path should be established prior to the expiration of the previous

one. The aim is to ensure that no packet will be in-flight when the path becomes invalid. To this end, a satellite $v_{i}$ initiates the path discovery process for a destination satellite at time rt_path_expiration_time - 
expiration_threshold, where rt_path_expiration_time is the value of the entry regarding destination satellite, which is stored in table $R T_{i}$.

Each path discovery process may fail to determine a path to destination. Since the existence of at least one path within the restricted route request area is guaranteed, failure to discover a path is the result of dropping RREQ or RREP packets due to congestion. In this case the path discovery process is repeated periodically until a path is found.

\section{Simulation Model}

The experiments conducted in this work aim at evaluating the performance of the proposed routing scheme. Since LAOR provides traffic adaptivity, its performance is compared to traffic adaptive schemes. Such schemes have been proposed only for centralized, periodic algorithms. In particular, three representative traffic adaptive routing schemes proposed in the literature so far [9],[10] are used for comparison. The simulation tool that was used for this purpose was developed in the platform of the detailed simulation model of ns2 [17]. The satellite extensions of the latter allow creating a framework for studying the performance of medium access control, routing and transport protocols. As far as routing in LEO satellite constellations is concerned, there exists only an implementation of a centralized Dijkstra algorithm, which only takes propagation delay into account. For the needs of our experiments we modified the implementation of the built-in centralized routing algorithm so that it can take account of queuing delay as well. Regarding the LAOR protocol, two versions were simulated. One that takes only propagation delay into account (non-traffic adaptive) in order to compute the shortest paths and another that is based on the total end-to-end delay (traffic-adaptive), that is the sum of propagation and queuing delays.

The five protocols were tested in an Iridium-like constellation, which is the most representative of polar constellations and is usually examined in bibliography. In this constellation, ISLs are switched off when satellites cross the polar regions; the latter are defined by a latitude threshold ( $60^{\circ}$ for Iridium). The rest of the simulation parameters are presented in Table 1. In order to enhance the performance of proactive

Table 1: Simulation parameters

\begin{tabular}{|l|c|}
\hline$\sharp$ of orbits & 6 \\
\hline$\sharp$ satellites pre plane & 11 \\
\hline Satellite altitude & $780 \mathrm{~km}$ \\
\hline Inclination & $86.4^{\circ}$ \\
\hline Interplane separation & $31.6^{\circ}$ \\
\hline Min. elevation angle & $8.2^{\circ}$ \\
\hline Cross-seam ISLs & No \\
\hline$\sharp$ of ISLs & 2 intra-plane +2 inter-plane \\
\hline ISL latitude threshold & $150^{\circ}$ \\
\hline Up/downlink bandwidth & $10 \mathrm{Mb} / \mathrm{s}$ \\
\hline ISL bandwidth & $500 \mathrm{packets}$ \\
\hline ISL LL queue size & $3000 \mathrm{packets}$ \\
\hline LAOR queue size & $6050 \mathrm{sec}$ \\
\hline Simulation duration & \\
\hline
\end{tabular}

protocols, that is, of the centralized and periodic routing schemes, the update interval of routing tables was set to $10 \mathrm{sec}$. In addition to the periodic computation, the calculation of the shortest paths is also triggered 
whenever a change in the ISL topology occurs.

Traffic inserted into the network was generated by 200 earth stations which were distributed over the six continents according to the hot spot scenario described in [18]. It should be noted that this scenario is based on the distribution of web servers and is more realistic for the case that the satellite system is used as a backbone to Internet. Furthermore, since earth stations represent traffic aggregation points, their number can be considered adequate for representing a real-life scenario. To model the traffic from each earth station, the bulk of the studies in this field used Poisson arrival processes (exponential distribution for interarrival times). However, we used an exponential ON/OFF generator, which is a generalized version of the exponential process, in order to model traffic bursts. This approach is closer to a real-life scenario. Table 2 tabulates the parameters of this traffic generation. According to Table 2, the chosen bitrates produce average (over both $\mathrm{ON}$ and $\mathrm{OFF}$ periods) bitrate values that range from 50 to $300 \mathrm{~kb} / \mathrm{s}$ per earth station. As a result the total traffic load offered to the system ranges from 10 to $60 \mathrm{Mb} / \mathrm{s}$ which can be considered reasonable for a real-life scenario. Higher values of offered load are not considered in this study since simulations indicate that they produce significant saturation to several parts of the network. It is clear, however, that the ISL bandwidth affects this situation. The chosen value for ISL bandwidth is 100 times greater than the scaled down value usually used in the literature [9], [10], [18]. This approach was adopted in order to implement a more realistic system and avoid the scaling down of ISLs bandwidth. As far as LAOR is concerned and for

Table 2: Traffic generator's parameters

\begin{tabular}{|l|c|}
\hline Packet size & 1500 bytes \\
\hline "On"period & $0.3 \mathrm{sec}$ \\
\hline "Off"period & $0.9 \mathrm{sec}$ \\
\hline bitrate during "On"periods & $200 \mathrm{~kb} / \mathrm{s}-1200 \mathrm{~kb} / \mathrm{s}$ \\
\hline
\end{tabular}

the needs of the presented simulation study, the size of RREQ packets was 52 bytes while the size of RREP packets was 48 bytes. This breaks down to 2 bytes for the pkt_type_id field, 8 bytes for the fields containing sequence numbers, 4 bytes for every other field and a link-layer header of 14 bytes. Obviously, the choice of field sizes represents the worst case and underestimates the performance of LAOR in terms of byte overhead.

\subsection{Link Cost Metrics}

In this subsection we comment on the different link-cost metrics that the examined routing protocols employ. As far as LAOR is concerned, according to Section 3.2 there are two options. LAOR may use only propagation delay as the link-cost metric or it can also take the queuing delay into account. In both cases, the delay that LAOR records in each link (propagation and, if needed, queuing) is the one encountered by the RREQ packet during its travel to the destination. On the other hand, the link-cost function used by the centralized-periodic routing protocols is based on a combination of propagation and queueing delays and is given by [9],[10]

$$
\text { Link cost }=W F_{\mathrm{PD}} \times P D+W F_{\mathrm{QD}} \times Q D
$$

where $P D$ and $Q D$ denote the propagation delay and queuing delay respectively, while $W F_{\mathrm{PD}}$ and $W F_{\mathrm{QD}}$ represent the weighting factors given to them. The first of these three protocols is based only on propagation delay, thus $W F_{\mathrm{PD}}$ was set to 1 and $W F_{\mathrm{QD}}$ to 0 . The remaining two protocols take queuing delay into account as well. In [9],[10] different combinations of $W F_{\mathrm{PD}}$ and $W F_{\mathrm{QD}}$ were studied. For the sake of fairness, we set $W F_{\mathrm{PD}}=1$ and $W F_{\mathrm{QD}}=1$ because this combination yields the best performance. Furthermore, any other combination is superficial and does not accurately reflect the network state. Nonetheless, there exists a difference between these two protocols, which lies in the method according to which the queuing delay is 
estimated. In the first algorithm, queuing delay is taken into consideration by means of an average value over the update interval of routing tables. The second one uses an exponential forgetting function (EFF). Both methods are frequently used by researchers [19],[20]. Let us now focus our attention on the latter method. To derive a feasible methodology for estimating queueing delay we take advantage of the ability of Layer- 2 protocols to provide statistics related to the ISL utilization. Then, according to the $M / M / 1$ queuing model [21] the mean number of packets in a queue $M_{\mathrm{PQ}}$ can be estimated by

$$
M_{\mathrm{PQ}}=\frac{u_{I S L}}{1-u_{I S L}}
$$

where $u_{I S L}$ is the mean link utilization provided by link layer (LL). When EFF is employed, link utilization is calculated as follows ${ }^{1}$ :

$$
u_{I S L}^{k}=l i n k \_s t a t e+d e c a y \cdot\left(u_{I S L}^{k-1}-l i n k \_s t a t e\right)
$$

where $u_{I S L}^{k}$ and $u_{I S L}^{k-1}$ are the estimated link utilizations when the $k^{t h}$ and $(k-1)^{t h}$ packets arrive at the interface queue in the current time interval. Concerning link state, it is set to 0 if there is no packet either in the interface queue or under transmission, otherwise it is set to 1 . The parameter decay denotes the forgetting rate and is defined as:

$$
\text { decay }=e^{\Delta t_{k}}
$$

where $\Delta t_{k}$ is the time interval between the arrivals of packets $k$ and $k-1$ in the queue. Then, the queuing delay can readily be estimated by the following expression:

$$
\text { Queuing delay }=M_{\mathrm{PQ}} \frac{\text { Packet size }}{\text { ISL throughput }}
$$

where the fraction denotes the packet transmission delay.

\subsection{Performance Metrics}

To evaluate the performance of the examined protocols, four performance metrics are used:

- mean end-to-end delay: the average time needed by the algorithm to successfully deliver packets to their destination. This metric directly evaluates the ability of the algorithm to provide shortest paths.

- mean delay jitter: the average delay variation experienced by two consecutive data packets. This metric provides an assessment of the algorithm's stability.

- end-to-end packet delivery ratio: the ratio of data packets delivered to the data packets generated. Hereafter, we will refer to this metric as delivery ratio.

- normalized routing overhead: the number of routing packets transmitted per data packet delivered (packet overhead). Another more meaningful metric is represented by the ratio of the total number of bytes related to routing packets to the total number of bytes of data packets (byte overhead).

In general, the first and the third metrics are reckoned to be the most important performance indicators when evaluating a routing protocol. However, when it comes to streaming applications, such as VoIP (telephony) and audio/video streaming, mean delay jitter is of paramount importance and should be kept to a minimum. Last but not least, despite the vast literature on routing in non-GEO satellite systems, the acute issue of routing overhead brought about by the exchange of information among satellites has scarcely been addressed. The fourth metric shows how efficiently the system resources are utilized and provides an assessment of the protocol ability to scale.

\footnotetext{
${ }^{1}$ This is the built-in method in ns2.
} 


\section{Simulation Results and Discussion}

In this section we present an extensive simulation study of the proposed protocol performance. The study comprises three sets of experiments. The first set is devoted to the comparison of LAOR with the routing schemes mentioned in the previous section. For the sake of clarity, these protocols are presented below, along with the notations used in the rest of the paper.

- Central.-PD: the centralized-periodic routing scheme when the link-cost metric is propagation delay.

- Central.-AV: the centralized-periodic routing scheme when the link-cost metric is the sum of propagation delay and average queuing delay.

- Central.-EXP: the centralized-periodic routing scheme when the link-cost metric is the sum of propagation delay and the queuing delay perceived by the exponential forgetting function.

- $\boldsymbol{L A O R - P D :}$ : the LAOR protocol when the link-cost metric is propagation delay.

- LAOR-TD: the LAOR protocol when the link-cost metric is the sum of propagation and queuing delay.

The five protocols are compared in terms of the metrics described in Section 4.2 for various traffic generation rates. The second experiment explores the effect of the parameter width in the performance of LAOR. Finally, the third experiment investigates the impact of bursty traffic on the performance of both LAOR and centralized schemes. At this point it should be noted that the presented results represent average values over 10 independent simulation runs. That number of runs provided $99 \%$ confidence intervals of $\pm 4 \%$ at worst.

\subsection{Performance vs traffic load}

This set of experiments represents the fundamental comparison of LAOR with periodic routing schemes. For this set of comparisons, the parameters expiration_threshold and width of the LAOR protocol were set to $0.5 \mathrm{sec}$ and 1 respectively. The rationale behind the selection of an expiration_threshold equal to $0.5 \mathrm{sec}$ is to ensure that no packets will be in-flight when the path will become invalid. Recall also that width determines the boundaries of the restricted route request area with respect to the y-axis. For the Iridium system, the minimum value that can be used for width is $w_{\min }=1$.

Figure 6 depicts mean end-to-end delay versus terminal's bitrate. It becomes evident from this figure that the LAOR protocol constitutes a significant improvement on periodic schemes. A stunning amelioration is observed for high bitrates. In particular, for bitrates higher than $1000 \mathrm{~kb} / \mathrm{s}$ mean end-to-end delay is dropped down by half. It must be noted that the performance of routing algorithms is more important in conditions of high system loading, since each system is desired to maximize the use of its resources. Such high loading conditions are represented by bitrates greater than $1000 \mathrm{~kb} / \mathrm{s}$. At the same time, in such conditions the overall offered traffic to the system is 50 to $60 \mathrm{Mb} / \mathrm{s}$. The striking results of the LAOR protocol are ascribed to its ability to capture traffic variations as well as changes in the propagation delay of ISLs, which stems from the fact that LAOR makes routing decisions upon request. As a result, the adaptation to the network state is smoother. Indeed, the LAOR protocol yields an enhanced performance compared to the three periodic routing schemes, even when the link-cost metric is propagation delay. On the other hand, when queuing delay is taken into account, the ability of LAOR to derive a path upon request, leads to the distribution of traffic to multiple almost equivalent paths. Another approach in achieving the same goal would be to calculate offline the $k$ best paths based on only propagation delay and then split the traffic among these paths. However, this approach introduces further complexity regarding the determination of the best $k$ for each constellation 


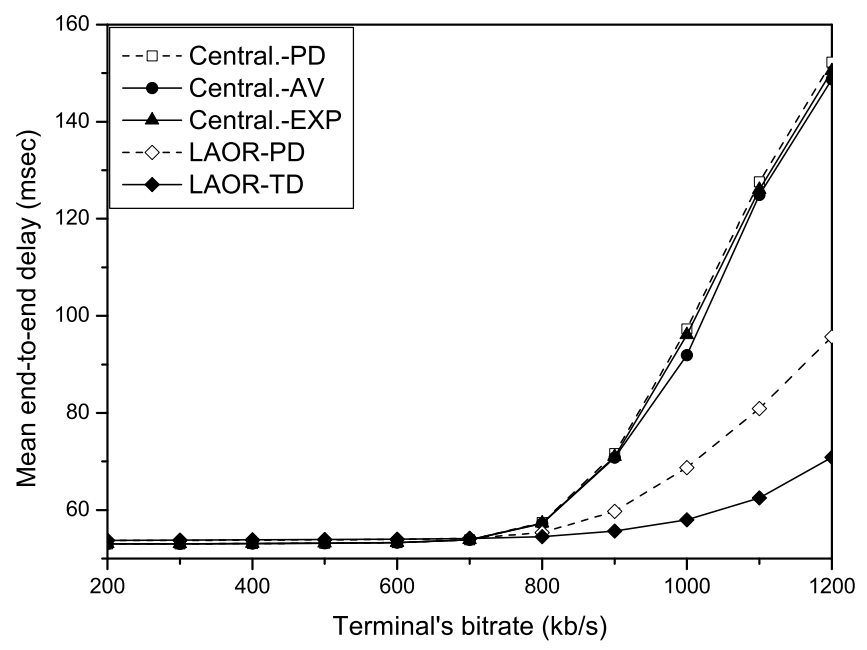

Figure 6: Mean end-to-end delay vs terminal's bitrate

and the maintenance of state for each path. The latter is of major importance since parts of a path may be used by several origin/destination pairs. Therefore, the timely collection of path information is required and involves the tradeoff between accuracy and overhead. However, LAOR, through its on-demand operation, provides smooth adaptation to the network state with low overhead. Another interesting result is that there exist no differences in the performance of the three periodic routing schemes, albeit one would expect the schemes that take account of queuing delay to outperform the one that relies only on propagation delay. This behavior can be explained by the fact that as soon as a new path is computed for a pair of satellites, all communications between terminals served by these two satellites will be forced to use this new path, hence making it congested. This phenomenon is repeated in every update cycle and is known as oscillation. It is more intense in connectionless communications, such as IP forwarding, since data flows quickly adapt to path switching.

The performance of the protocols in terms of mean delay jitter is illustrated in figure 7. As expected, LAOR outperforms centralized schemes. An amelioration in network performance is witnessed even for moderate bitrates regardless of the employed link-cost metric. Not only do these results prove that LAOR succeeds in finding the less congested path, but that successive calculated paths, located within the restricted route request area, do not differ significantly in terms of end-to-end delay as well. This is an indication that LAOR effectively splits traffic into different paths. Of course, when the link cost metric is based only on propagation delay the performance slightly aggravates. At the other extreme, periodic protocols suffer from the effect of oscillations. The protocol that relies only on propagation delay achieves a better performance than the other two protocols since oscillations are not so intense in this case. On the contrary, oscillations are mostly the result of queueing delay variations. Furthermore, Central-EXP and Central-AV, in order to minimize total delay, result in paths that consist of a greater number of hops. As we have already explained, these paths become congested thereupon their establishment, thus variations in end-to-end delay are slightly higher.

Our next objective is to show that LAOR can accurately deliver packets to their destination regardless of the traffic intensity. Table 3 provides a tabulation of the delivery ratios for all the examined schemes and for different values of the terminal's bitrate. Although all the examined routing schemes manage to successfully deliver generated packets to their destination for all moderate bitrates, when bitrate increases to $900 \mathrm{~kb} / \mathrm{s}$ and above, some links become congested and therefore, packets are dropped. At this point it should be mentioned that the values of the delivery ratio depend heavily on the chosen LL queue size. The latter determines the 


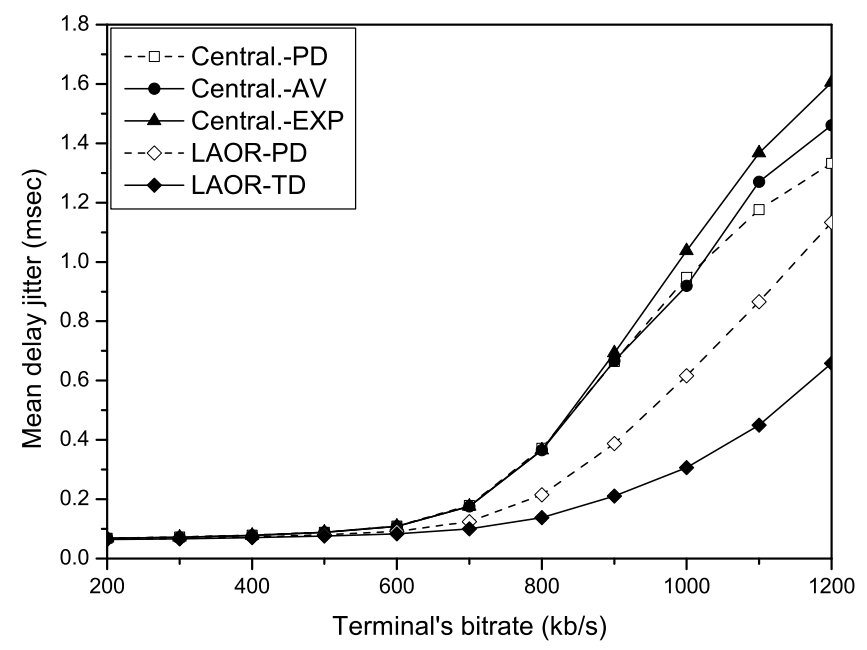

Figure 7: Mean delay jitter vs terminal's bitrate

Table 3: Delivery Ratio

\begin{tabular}{|l|c|c|c|c|c|}
\hline Bitrate $(\mathrm{kb} / \mathrm{s})$ & Central.-PD & Central.-AV & Central.-EXP & LAOR-PD & LAOR-TD \\
\hline $200-700$ & 1 & 1 & 1 & 1 & 1 \\
\hline 800 & 0.999967 & 0.999962 & 0.999967 & 0.999990 & 0.999992 \\
\hline 900 & 0.999115 & 0.999122 & 0.999162 & 0.999904 & 0.999986 \\
\hline 1000 & 0.995494 & 0.995826 & 0.995662 & 0.999346 & 0.999932 \\
\hline 1100 & 0.988451 & 0.988860 & 0.988717 & 0.998021 & 0.999731 \\
\hline 1200 & 0.979689 & 0.980435 & 0.980061 & 0.995544 & 0.999019 \\
\hline
\end{tabular}

trade-off between queueing delay and packet drop rate. If LL queue size is decreased, then it is obvious that the delivery ratio will decrease due to the increased drops in ISL queues. Centralized schemes are affected in a greater extent because they suffer by increased congestion in ISL queues. This is confirmed by the increased delay of centralized schemes compared to LAOR. On the other hand, if LL queue size is increased, the delivery ratio for both algorithms may reach up to $100 \%$. However, the end-to-end delay will increase since packets are delayed rather than dropped. Again centralized schemes are affected the most, since longer queues are formed. The aim of comparing the delivery ratio is to exhibit the tendency of the algorithms in avoiding congested points in the network. Nonetheless, a delivery ratio around $98 \%$ may be considered low depending on the service requirements and the volume of delivered traffic. It should be emphasized that in the case of LAOR, packets can also be dropped while waiting in the LAOR queue for a path to be established. However, the delivery ratio of the LAOR protocol is higher than the one of the centralized routing schemes. Note that LAOR-TD outperforms the rest of the schemes, though the performance of LAOR-PD is very close. In addition, the algorithms that take account of queuing delay perform better than those that rely only on propagation delay. Concerning the periodic routing schemes, their poor performance compared to LAOR can be attributed to the limited ability of the periodic mechanism to effectively estimate the network state. Thereby, this explains why these schemes perform poorly in terms of end-to-end delay and delay jitter.

Figure 8 presents the mean number of hops per connection. It is obvious that all the schemes that use a link-cost metric that consists of both propagation and queuing delays compute paths that comprise more hops. Under further scrutiny, we observe that the mean number of hops of the Central.-EXP scheme is somewhat greater than Central.- $A V$ 's, hence the larger mean delay jitter of the former. As regards the mean 
number of hops of $L A O R-T D$, simulation results reveal that low queuing delay is the decisive factor for path discovery in congested areas of the network. This is apparent from the greater mean number of hops that this scheme presents. Moreover, as bitrate increases so does the mean number of hops, which means that the protocol routes traffic through longer (in terms of hops) but less congested paths. On the contrary, the average number of hops of the centralized protocols decreases as bitrate increases, which shows their inability to capture the current network state. This can also be explained from the results presented in Table 3. When traffic congestion occurs, the centralized schemes drop more packets than LAOR. Obviously, packets that are routed through shorter (in terms of hops) paths stand a greater chance of being delivered to their destination than packets that use longer links. In other words, a significant number of packets that are routed through long paths are dropped, hence the decrease in the mean number of hops as bitrate increases. In addition, the results concerning $L A O R-P D$ testify to the protocol's ability to successfully route packets irrespective of the network traffic. In particular, only a few packets are dumped and the mean number of hops is not affected.

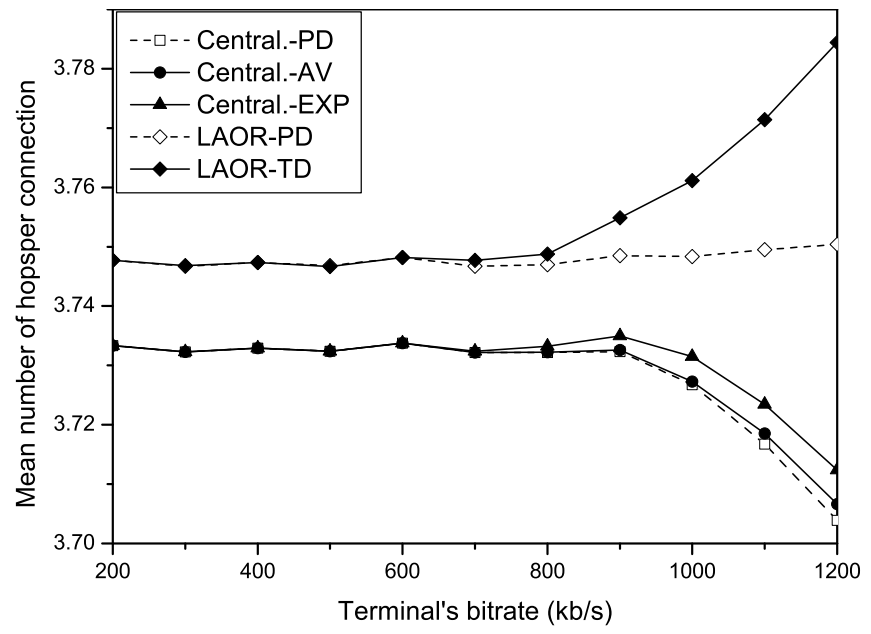

Figure 8: Mean number of hops vs terminal's bitrate

In order for our study to be complete we also had to evaluate the signaling overhead that LAOR induces into the system. Table 4 presents both the normalized packet overhead and the normalized byte overhead. Apparently, the overhead of this protocol is negligible regardless of the employed link-cost metric. Byte overhead appears to be much lower than packet overhead since RREQs and RREPs are much smaller than data packets. Even for the lowest bitrate, which represents the worst-case scenario in terms of routing overhead, the normalized byte overhead is less than $0.1 \%$, whereas packet overhead is smaller than $1 \%$. It should be noted that the two centralized protocols that take account of queuing delay have not been assessed because their performance in terms of overhead depends on the location of the central node which collects the required information from all satellites, computes the shortest paths, and disseminates the routing tables, as well as on the route table update interval. Nevertheless, supposing that a ground station is shouldered this daunting responsibility, we can come up with a rough estimate of the normalized packet overhead. To this end, let us assume that the algorithm is only periodically implemented every $10 \mathrm{sec}$. If we take into consideration that each satellite generates a packet with information about the queuing delay in its ISL queues, then we are led to the conclusion that every 10 sec 65 packets are generated (we do not consider the packet generated by the satellite that is directly connected to the designated ground station). As soon as the ground terminal computes the shortest paths it should distribute the routing tables to the satellites, thus 65 other packets are inserted into the network. This translates in 130 packets every 10 sec. Hence, the total amount of packets generated during the simulated time interval is $130 \cdot(6050 / 10)=96800$. The average number of delivered 
Table 4: Normalized Overhead

\begin{tabular}{|l|c|c|c|c|}
\hline \multirow{2}{*}{ Bitrate $(\mathrm{kb} / \mathrm{s})$} & \multicolumn{2}{|c|}{ LAOR-PD } & \multicolumn{2}{c|}{ LAOR-TD } \\
\cline { 2 - 5 } & Packet Overhead(\%) & Byte Overhead(\%) & Packet Overhead(\%) & Byte Overhead(\%) \\
\hline 200 & 0.9593626 & 0.0325039 & 0.9486526 & 0.032148 \\
\hline 300 & 0.6499853 & 0.0220204 & 0.6388396 & 0.0216494 \\
\hline 400 & 0.4921545 & 0.0166723 & 0.4808541 & 0.0162958 \\
\hline 500 & 0.3982082 & 0.0134892 & 0.3874941 & 0.0131321 \\
\hline 600 & 0.335055 & 0.0113487 & 0.3237726 & 0.0109727 \\
\hline 700 & 0.2890788 & 0.009791 & 0.2774186 & 0.0094018 \\
\hline 800 & 0.2556861 & 0.0086039 & 0.2441891 & 0.0082758 \\
\hline 900 & 0.2306593 & 0.0078107 & 0.2163541 & 0.0073324 \\
\hline 1000 & 0.2115723 & 0.0071633 & 0.1949158 & 0.0066058 \\
\hline 1100 & 0.1986213 & 0.0067237 & 0.1788703 & 0.0060621 \\
\hline 1200 & 0.1878121 & 0.0063566 & 0.1653855 & 0.0056052 \\
\hline
\end{tabular}

data packets for bitrate $=200 \mathrm{~kb} / \mathrm{s}$ is around $4.7 \cdot 10^{6}$, therefore, the normalized packet overhead is $0.02 \%$. This performance is better by a factor of 50 compared to the performance of LAOR. However, this can be considered an acceptable trade-off if the small absolute size of packet overhead (less than $1 \%$ in the worst case) for LAOR is considered in combination with its improved performance. Furthermore, it is reasonable to believe that in the case of the centralized protocols the size of routing packets would be greater than the one of RREQs and RREPs, since the former would have to contain much more information. For example, packets containing the results of route calculation should carry 65 paths, one for each destination. On this account, the byte overhead of centralized routing schemes is expected to be greater than the byte overhead of LAOR. Finally, as regards Central.-PD, it induces no overhead since it capitalizes on the deterministic dynamics of LEO networks. Nevertheless, this advantage is nullified by its poor performance.

\subsection{The impact of parameter width}

The next set of experiments aims at investigating the impact of the parameter width on the performance of the LAOR protocol. Thus, the LAOR protocol is evaluated for width $=1$ and 3 . In general, a wider restricted route request area translates into a greater chance of finding a less congested path. Of course, the link-cost metric that is considered is total end-to-end delay since it does not make sense to use a value larger than 1 (which is the minimum for Iridium) when the link cost metric is propagation delay. Moreover, we developed another variant of this protocol that adjusts the boundaries of the restricted route request area according to the virtual coordinates of the pair of communicating satellites, hereinafter referred to as adaptive. The function that calculates the boundaries on the $y$-axis for the adaptive version of LAOR, when implemented on the Iridium-like system, is as follows:

$$
\text { width }= \begin{cases}3, & \text { if }\left|\bar{y}_{s}-\bar{y}_{d}\right|=5 \\ 0, & \text { if }\left|\bar{x}_{s}-\bar{x}_{d}\right|=0 \text { and }\left|\bar{y}_{s}-\bar{y}_{d}\right|<5 \\ 1, & \text { in any other case }\end{cases}
$$

where $\left(\bar{x}_{s}, \bar{y}_{s}\right)$ and $\left(\bar{x}_{d}, \bar{y}_{d}\right)$ are the pairs of the virtual coordinates of the source and destination satellites respectively ${ }^{2}$. The aim of the adaptive scheme is to preserve the advantages that stem from a wider restricted route request area without increasing routing overhead. To this end, Eq. 12 allows two satellites, whose

\footnotetext{
${ }^{2}$ It should be noted that, according to Eq. 12, the value width $=0$ can be used for specific cases of origin/destination pairs. However, as mentioned before, when the same value of the parameter width should be used for any pair of communicating
} 


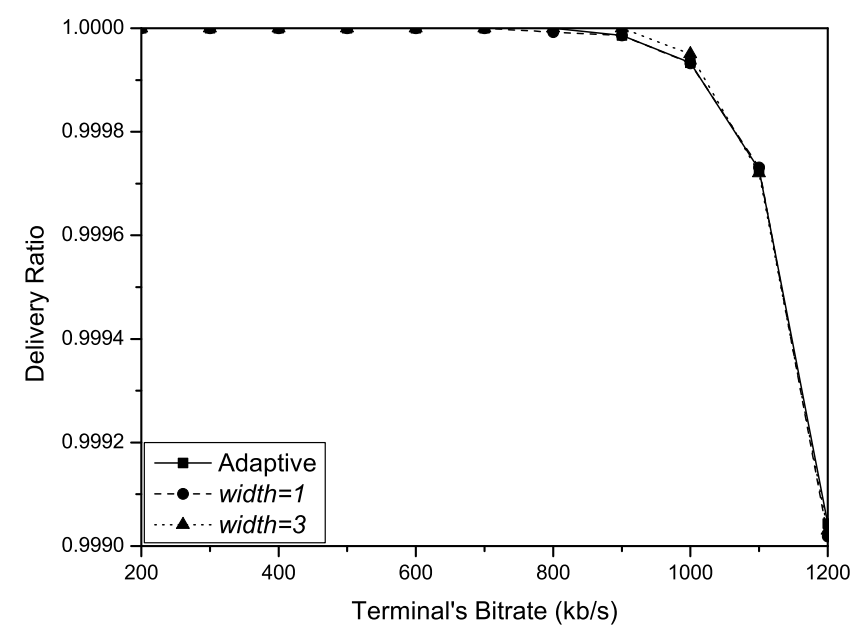

Figure 9: Delivery ratio vs terminal's bitrate

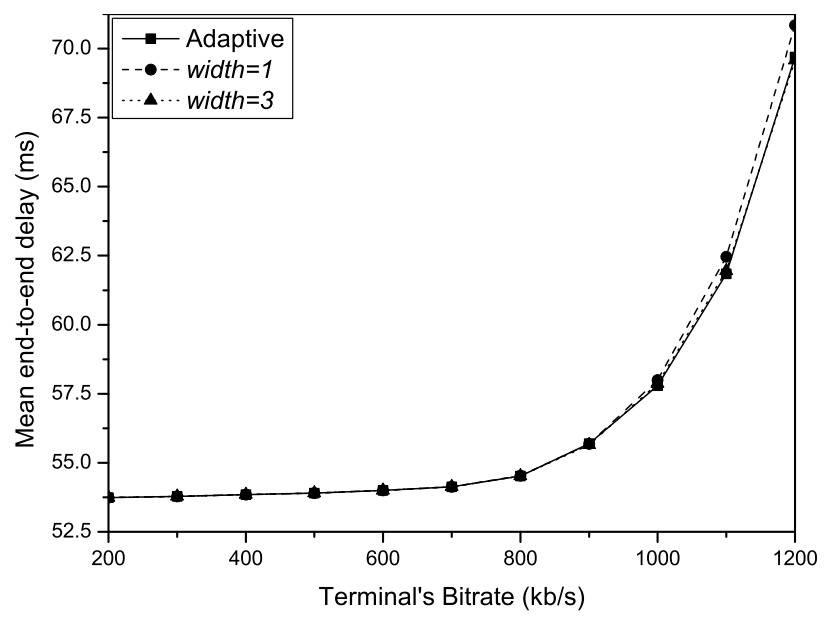

Figure 10: Mean end-to-end delay vs terminal's bitrate

distance on the $y$-axis is the maximum possible (that is, $\left|\bar{y}_{s}-\bar{y}_{d}\right|=5$ ), to communicate through a path either over the North pole or the South pole.

Figure 9 presents the delivery ratio versus the terminal's bitrate. As it can be seen in this figure, there do not exist significant performance disparities among the examined protocols. However, the scheme that uses width $=3$ performs slightly better since the wider restricted route request area allows it to find longer (in terms of hops) but less congested paths. Concerning the other two schemes, they present a similar performance. This fact reveals that for most of the cases in the adaptive scheme, the pair of communicating satellites is such that width $=1$. Figure 10 illustrates the mean end-to-end delay. It is evident that again the performance of the different protocols is similar. However, under further scrutiny one can observe that the best performance is attained when width $=3$. This can be explained by the fact that as the value of the parameter width increases, the algorithm achieves to establish a path that may consist of a greater number of hops, but it less congested than other alternative paths that are comprised of a smaller number of hops. Thus the queuing delay perceived by data packets is lower which in turn results in lower end-to-end delay. Regarding the adaptive scheme, it performs similarly to the scheme that uses the value width $=3$. Hence, this

satellites (i.e., the case of non-adaptive schemes), then the minimum value that guarantees path existence is 1 for an Iridium-like constellation. 


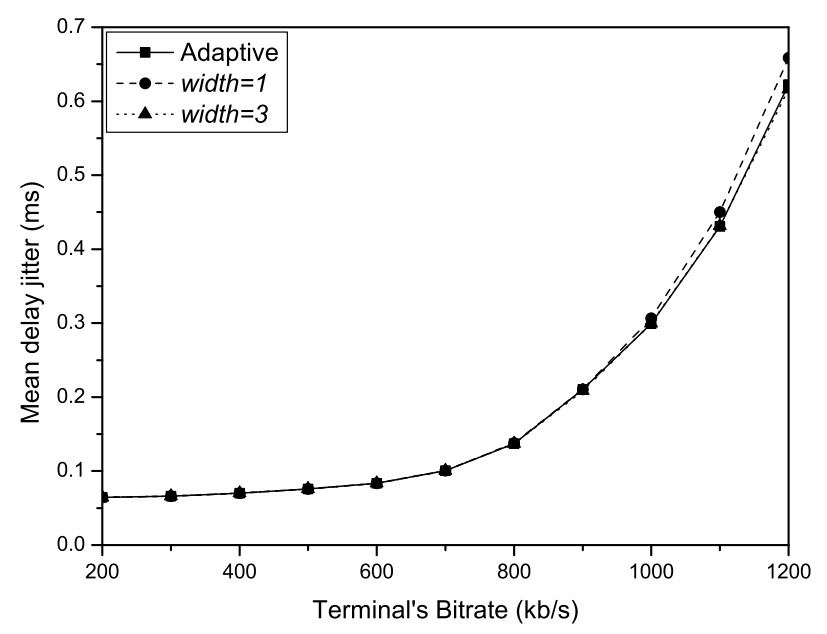

Figure 11: Mean delay jitter vs terminal's bitrate

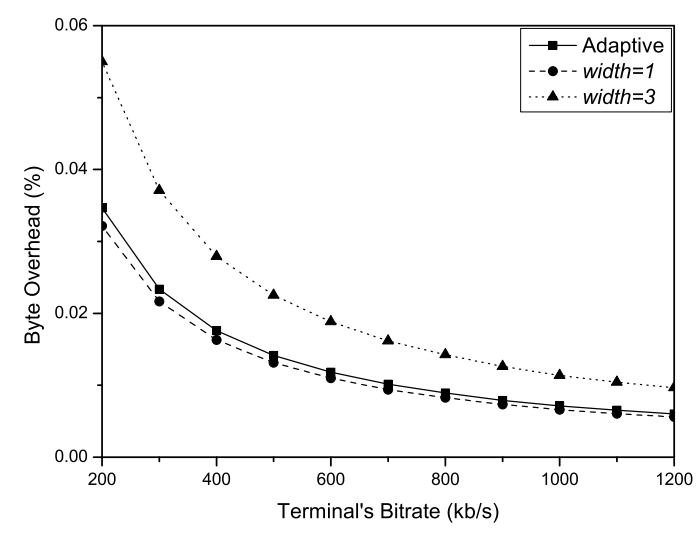

Figure 12: Packet overhead vs terminal's bitrate

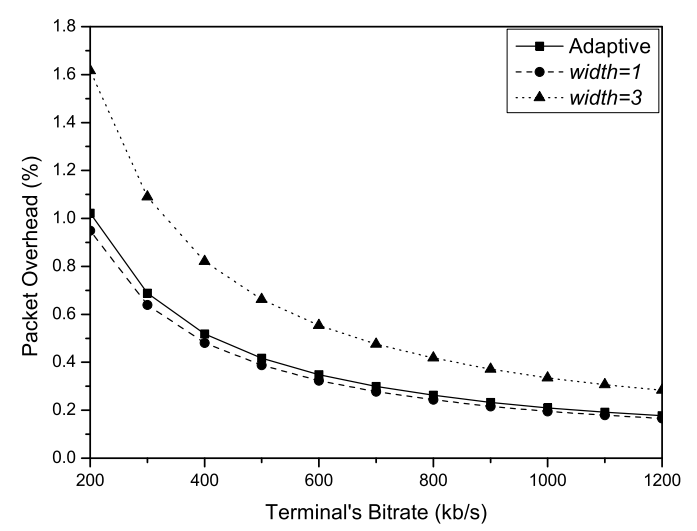

Figure 13: Byte overhead vs terminal's bitrate

means that the adaptive scheme can effectively adjust the restricted route request area based on the virtual coordinates of the communicating pair of satellites. Similar behaviors can be seen in figure 11, which depicts the mean delay jitter. The next set of figures illustrates the routing overhead. We expect that as the value of the parameter width increases, routing overhead will increase as well. Figure 12 depicts the normalized packet overhead, while figure 13 presents the normalized byte overhead. These figures substantiate our expectations. The best performance is attained by the scheme with width $=1$, the performance of the adaptive scheme is very close though. As expected, the scheme with width $=3$ presents the worst performance on account of the greater number of routing packets that are generated. Nevertheless, the packet and byte overheads are still low. From the last four figures we are led to the conclusion that the adaptive scheme attains the best overall performance, however the performance disparities among these three schemes are not really significant.

\subsection{Performance under bursty traffic}

In the third set of experiments, the $L A O R$-TD protocol, that is, the LAOR protocol when the link-cost metric is based on the total end-to-end delay, is compared to the Central.-AV protocol for different combinations of the ON/OFF periods of the traffic generator. The aim of this set of experiments is to demonstrate that the performance disparity between these two routing protocols is not affected by a change in the ON/OFF ratio of the traffic generator. The latter indicates how smoothly or abruptly traffic is inserted into the network, i.e., 


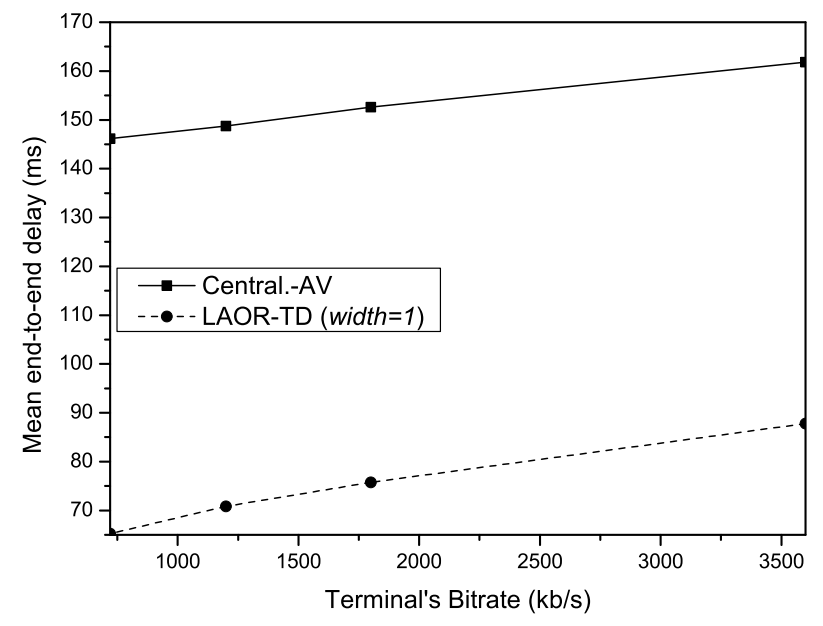

Figure 14: Mean end-to-end delay vs terminal's bitrate

how bursty the traffic is. Therefore in this set of experiments we vary the ON/OFF ratio while the average (over both ON and OFF periods) traffic inserted in the network per time unit is kept constant and equals $300 \mathrm{~kb} / \mathrm{s}$ per earth station. To achieve this we also vary the bitrate during ON periods of each station. Table 5 provides a tabulation of the parameters that were used in these experiments. Note that for all cases the

Table 5: Traffic generator's parameters

\begin{tabular}{|l|l|r|}
\hline ON Period & OFF Period & Bitrate during ON periods \\
\hline 0.5 & 0.7 & $720 \mathrm{~kb} / \mathrm{s}$ \\
\hline 0.3 & 0.9 & $1200 \mathrm{~kb} / \mathrm{s}$ \\
\hline 0.2 & 1.0 & $1800 \mathrm{~kb} / \mathrm{s}$ \\
\hline 0.1 & 1.1 & $3600 \mathrm{~kb} / \mathrm{s}$ \\
\hline
\end{tabular}

average traffic is $300 \mathrm{~kb} / \mathrm{s}$ per station. This value of average traffic equals the average traffic that was inserted in the network in all previous experiments when the bitrate during ON periods was set to $1200 \mathrm{~kb} / \mathrm{s}$ (see row 3 of Table 5). This case represents a heavily loaded network where the performance of routing algorithms becomes increasingly important. The results for lower values of the average traffic offered to the network are qualitatively similar to the results that will be presented in this section.

Figure 14 presents the first metric of interest, that is mean end-to-end delay. As expected, the LAOR protocol outperforms the centralized routing protocol for any ON/OFF combination. It is interesting to notice the impact of the ON/OFF ratio on mean end-to-end delay. When the ON period increases, therefore the OFF period correspondingly decreases, the mean end-to-end delay reduces. The main reason for this behavior is that small ON periods model bursts in traffic, therefore more packets are accumulated in ISL queues. The latter translates in higher queuing delay, hence higher end-to-end delay. It should also be noted that the mean end-to-end delay of the periodic protocol is twice the one of the LAOR protocol. The mean delay jitter is illustrated in figure 15. As it becomes apparent from this figure, the LAOR protocol manages to keep this important performance metric to a minimum. In general, as explained earlier, the proposed protocol succeeds in finding the shortest path, namely the less congested path. Therefore variations in the queuing delay are not really significant, thereby mean delay jitter diminishes. The results presented in figure 16 reveal another positive characteristic of the LAOR protocol. While the periodic protocol drops more packets 


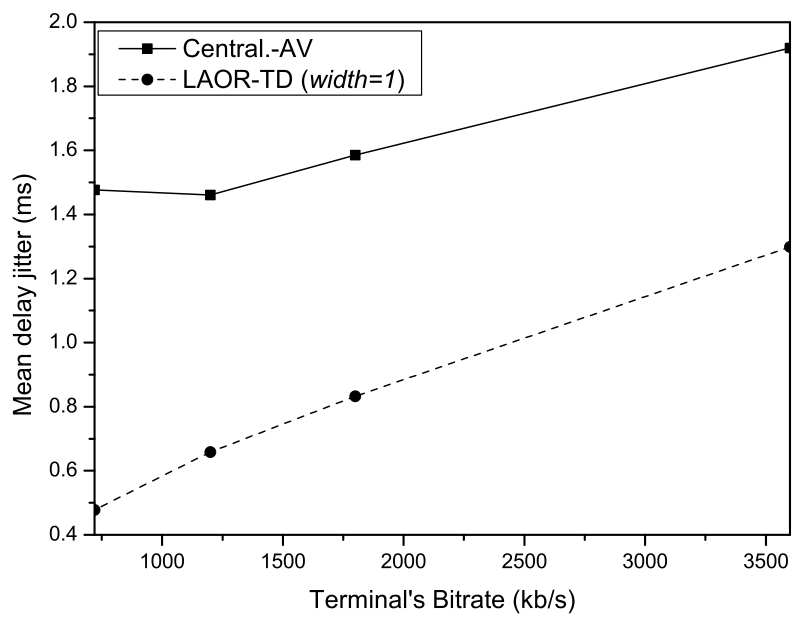

Figure 15: Mean delay jitter vs terminal's bitrate

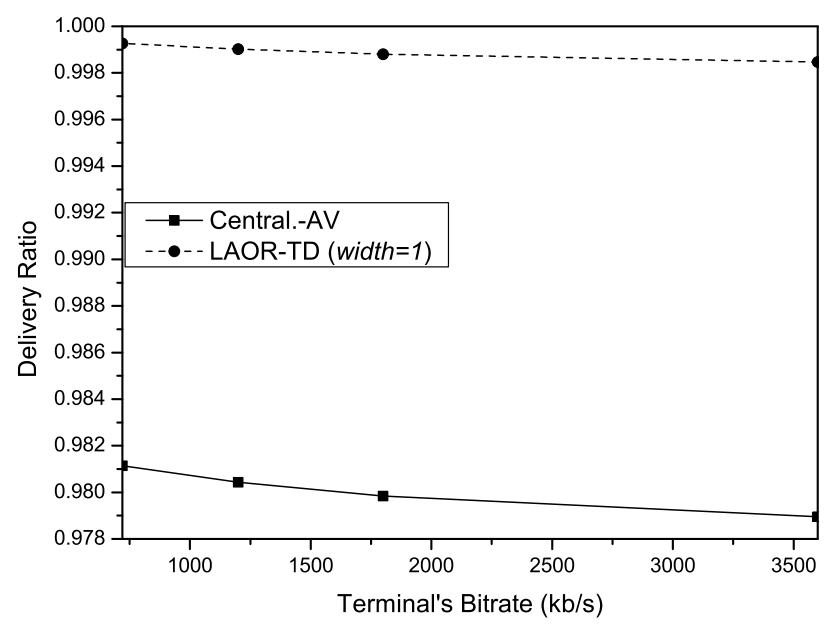

Figure 16: Delivery ratio vs terminal's bitrate

as the ON period reduces (burst size increases), the LAOR protocol appears to be immune to such a decrease. This is due to the ability of the latter to evade congested network areas. As previously stated, when the ON period decreases, the number of queued packets increases and so does the probability that a packet will be dropped. The periodic protocol fails to split the traffic into different paths, therefore congestion occurs in many ISLs. On the other hand, the LAOR protocol achieves a better ISL utilization, which in turn results in less loaded ISLs. Finally, as far as routing overhead is concerned, simulation results indicate that both normalized packet and normalized byte overhead are around $0.16 \%$ and $0.0055 \%$ respectively for all the examined ON/OFF ratios. As far as the centralized-periodic routing protocol is concerned, a rough estimation of the packet overhead was given earlier. Moreover, it should be noted that the routing overhead of the centralized protocol is not affected by the ON/OFF ratio since each satellite periodically sends one packet to a designated ground station. Therefore, the routing overhead imposed by LAOR is at least comparable to the best-case routing overhead induced by the centralized-periodic protocols. Furthermore, as explained 
previously and proved by figure 16, more routing packets will be dropped in the case of the centralized protocol due to increased congestion. Consequently, more routing packet retransmissions will be performed, resulting in an increase of routing overhead.

\section{Conclusions}

In this work we proposed and evaluated the performance of a Location-Assisted On-demand Routing (LAOR) protocol for LEO satellite networks. LAOR introduces for the first time in satellite networks the concept of on-demand routing. According to this strategy, the shortest path discovery procedure is invoked independently for each individual communication request to provide better estimation of the network state and avoid congested areas. Furthermore, in order to diminish the signaling overhead induced in the system, a mechanism has also been proposed that aims to reduce the number of satellites that will take part in the aforementioned procedure by utilizing the deterministic dynamics of satellite movement. The proposed protocol was compared to three centralized routing schemes that compute the shortest paths periodically based on the Dijkstra algorithm. The good characteristics of the LAOR protocol were corroborated by ample simulation experiments, where significant gains in performance were witnessed for all scenarios examined. Specifically, LAOR was shown to attain much lower end-to-end delay, delay-jitter and higher delivery ratio with negligible overhead. This fact renders it an excellent choice for future LEO satellite networks.

\section{References}

[1] G. Maral, M. Bousquet, Satellite Communications Systems: Systems, Techniques and Technology, $2^{\text {nd }}$ Edition, John Wiley \& Sons, 1993.

[2] A. Jamalipour, Low Earth Orbital Satellites for Personal Communication Networks, Artech House, Boston-London, 1998.

[3] B. Evans, M. Werner, E. Lutz, M. Bousquet, G. E. Corazza, G. Maral, R. Rumeau, E. Ferro, Integration of Satellite and Terrestrial Systems in Future Multimedia Communications, IEEE Wireless Communications 12 (5) (2005) 72-80.

[4] T. Taleb, N. Kato, Y. Nemoto, Recent Trends in IP/NGEO Satellite Communication Systems: Transport, Routing, and Mobility Management Concerns, IEEE Wireless Communications 12 (5) (2005) $63-69$.

[5] C. E. Perkins, S. R. D. Elizabeth M. Royer, Ad hoc on-demand distance vector (AODV) routing, RFC 3561 (July 2003).

URL http://www.ietf.org/rfc/rfc3561.txt

[6] M. Werner, A Dynamic Routing Concept for ATM-Based Satellite Personal Communication Networks, IEEE J. Select. Areas Commun. 15 (8) (1997) 1636-1648.

[7] M. Werner, C. Delucchi, H. J. Vögel, G. Maral, J.-J. D. Ridder, ATM-Based Routing in LEO/MEO Satellite Networks with Intersatellite Links, IEEE J. Select. Areas Commun. 15 (1) (1997) 69-82.

[8] H. Uzunalioğlu, I. F. Akyildiz, M. D. Bender, A routing algorithm for connection-oriented Low Earth Orbit (LEO) satellite networks with dynamic connectivity, Wireless Networks 6 (111) (2000) 181-190. 
[9] M. Mohorcic, A. Svigelj, G. Kandus, M. Werner, Performance evaluation of adaptive routing algorithms in packet-switched intersatellite link networks, Int. Journal of Satellite Communications 20 (2) (2002) $97-120$.

[10] M. Mohorcic, M. Werner, A. Svigelj, G. Kandus, Adaptive routing for packet-oriented intersatellite link networks: performance in various traffic scenarios, IEEE Trans. Wireless Commun. 1 (4) (2002) 808-818.

[11] I. F. Akyildiz, E. Ekici, M. D. Bender, MLSR: A Novel Routing Algorithm for Multilayered Satellite IP Networks, IEEE/ACM Trans. Networking 10 (3) (2002) 411-424.

[12] H. S. Chang, B. W. Kim, C. G. Lee, S. L. Min, Y. Choi, H. S. Yang, D. N. Kim, C. S. Kim, FSA-Based Link Assignment and Routing in Low-Earth Orbit Satellite Networks, IEEE Trans. Veh. Technol. 47 (3) (1998) 1037-1048.

[13] I. Gragopoulos, E. Papapetrou, F.-N. Pavlidou, Performance Study of Adaptive Routing Algorithms for LEO Satellite Constellations under Self-Similar and Poisson Traffic, Space Communications 16 (1) (2000) 15-22.

[14] E. Papapetrou, F.-N. Pavlidou, A Proposal of Optimal Routing Techniques for non-GEO Satellite Systems, International J. of Wireless Inform. Networks 8 (2) (2001) 75-83.

[15] E. Ekici, I. F. Akyildiz, M. D. Bender, A Distributed Routing Algorithm for Datagram Traffic in LEO Satellite Networks, IEEE/ACM Trans. Networking 9 (2) (2001) 137-147.

[16] E. M. Royer, C.-K. Toh, A review of current routing protocols for ad hoc mobile wireless networks, IEEE Personal Communications 6 (2) (1999) 46-55.

[17] K. Fall, K. Varadhan, The ns manual, VINT Project, Univ. California, Berkeley, CA (2001). URL http://www.isi.edu/nsnam/ns/ns-documentation.html

[18] M. Mohorcic, A. Svigelj, G. Kandus, Y. F. Hu, R. E. Sheriff, Demographically weighted traffic flow models for adaptive routing in packet-switched non-geostationary satellite meshed networks, Comput. Networks 43 (2) (2003) 113-131.

[19] P. Gupta, P. R. Kumar, A system and traffic dependent adaptive routing algorithm for ad hoc networks, in: Proceedings of the 36th IEEE Conference on Decision and Control, San Diego, California, 1997, pp. 2375-2380.

[20] S. Lohier, S.-M. Senouci, Y. Ghamri-Doudane, G. Pujolle, A reactive qos routing protocol for ad hoc networks., in: EUSAI, 2003, pp. 27-41.

[21] D. Bertsekas, R. Gallager, Data networks (2nd ed.), Prentice-Hall, Inc., Upper Saddle River, NJ, USA, 1992. 